\title{
Supporting people who have eating and drinking difficulties
}

\author{
Authors: Karen Porter, ${ }^{\mathrm{A}}$ Nicola Burch, ${ }^{\mathrm{B}}$ Claire Campbell, ${ }^{\mathrm{C}}$ Chris Danbury, ${ }^{\mathrm{D}}$ Charles Foster, ${ }^{\mathrm{E}}$ Simon Gabe, ${ }^{\mathrm{F}}$ \\ Andrew Goddard, ${ }^{G}$ Katie Harp, ${ }^{\mathrm{H}}$ Anne Holdoway, ${ }^{\mathrm{I}}$ Tom Hughes, ${ }^{\mathrm{J}}$ Karen Le Ball, ${ }^{\mathrm{K}}$ Jeremy Nightingale, \\ Andrew Rochford, ${ }^{\mathrm{M}}$ Alex Ruck Keene, ${ }^{\mathrm{N}}$ Alison Smith, ${ }^{\mathrm{O}}$ Trevor Smith ${ }^{\mathrm{P}}$ and Aminda De Silva ${ }^{\mathrm{Q}}$
}

Eating and drinking are essential for maintenance of nutrition and hydration, but are also important for pleasure and social interactions. The ability to eat and drink hinges on a complex and coordinated system, resulting in significant potential for things to go wrong.

The Royal College of Physicians (RCP) has published updated guidance on how to support people who have eating and drinking difficulties, particularly towards the end of life.

Decisions about nutrition and hydration and when to start, continue or stop treatment are some of the most challenging to make in medical practice. The newly updated guidance aims to support healthcare professionals to work together with patients, their families and carers to make decisions around nutrition and hydration that are in the best interests of the patient. It covers the factors affecting our ability to eat

Authors: ${ }^{\text {A }}$ editorial manager, Royal College of Physicians, London, UK; ${ }^{B}$ consultant gastroenterologist, University Hospital Coventry, Coventry, UK; ${ }^{C}$ nutrition clinical nurse specialist, Frimley Health NHS Foundation Trust, Frimley Park, UK; ${ }^{\mathrm{D}}$ consultant in anaesthetics and intensive care, Royal Berkshire NHS Foundation Trust, Reading, UK; Efellow, Green Templeton College, Oxford, UK and barrister, 4-5 Gray's Inn Square, London, UK; F consultant gastroenterologist, London North West University Healthcare NHS Trust, London, UK; ${ }^{G}$ consultant gastroenterologist, University Hospitals of Derby and Burton NHS Foundation Trust, Derby, UK and president, Royal College of Physicians, London, UK; ${ }^{\mathrm{H}}$ Clinical lead speech and language therapist, Royal Hospital for Neurodisability, London, UK; I consultant dietitian, BMI Bath Clinic, Bath, UK, Dorothy House Hospice, Bradford-on-Avon, UK and Royal United Hospitals Bath NHS Foundation Trust, Bath, UK; ${ }^{3}$ consultant neurologist, Cardiff and Vale University Health Board, Cardiff, UK; K consultant geriatrician, NHS Highland, Inverness, UK; ' ${ }^{\text {consultant }}$ gastroenterologist, London North West University Healthcare NHS Trust, London, UK; ${ }^{\mathrm{M}}$ consultant gastroenterologist, Barts

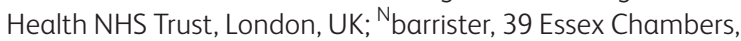
London, UK and visiting professor, Dickson Poon School of Law,

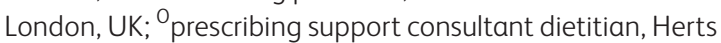
Valleys Clinical Commissioning Group, Hemel Hempstead, UK; ${ }^{P}$ consultant gastroenterologist, University Hospital Southampton NHS Foundation Trust, Southampton, UK and president, British Association for Parenteral and Enteral Nutrition, Redditch, UK;

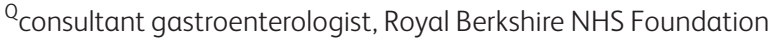
Trust, Reading, UK and chair of the working party and drink, strategies to support oral nutrition and hydration, techniques of clinically-assisted nutrition and hydration, and the legal and ethical framework to guide decisions about giving and withholding treatment, emphasising the two key concepts of capacity and best interests.

This article aims to provide an executive summary of the guidance.

KEYWORDS: eating and drinking difficulties, dietary modification, clinically-assisted nutrition and hydration, end-of-life care, ethical decisions

DOI: 10.7861/clinmed.2021-0161

\section{Introduction}

Over the past 2 decades, we have seen a dramatic change in patient populations in our hospitals, with an older demographic who have increasingly complex multiple long-term conditions, including cerebrovascular disease, degenerative neurological diseases and frailty. There are currently 885,000 people with dementia in the UK, and this is projected to rise to 1.6 million by 2040. ${ }^{1}$

Difficulties with eating and drinking are common in these populations and the challenges they present to all concerned are considerable. As well as weight loss and dehydration, problems with eating and drinking may be associated with very serious physical complications including episodic laryngeal obstruction, aspiration into the trachea and bronchi, secondary pulmonary infection, and bronchiectasis.

The distress (for the patient and family) of bouts of coughing and choking at mealtimes and the resulting disruption of domestic routine, social isolation and fear of eating and drinking may have a profound effect on the confidence and wellbeing of the patient and their family, and can be a significant source of anxiety and distress.

New guidance from the Royal College of Physicians (RCP) updates the previous Oral feeding difficulties and dilemmas published in 2010, particularly in relation to recent changes in the law governing procedures for the withdrawal of clinicallyassisted nutrition and hydration (CANH) and other life-sustaining treatments. ${ }^{2,3}$

It aims to outline what constitutes best practice within the existing legal framework, to enable healthcare professionals to fulfil their responsibilities to the patient and their family, focusing 
on the best interests of the patient. Updated throughout, it includes a new chapter on dietary modifications and a series of illustrative examples of patients to help guide practice. ${ }^{2}$

The guidance is primarily for medical and healthcare professionals, particularly those involved in caring for people who have eating and drinking difficulties, including gastroenterologists, ward nurses, geriatricians, dietitians, speech and language therapists, neurologists, palliative care teams, and care home and community nurses.

This summary covers the following areas in brief; more detail can be found in the full guidance: ${ }^{2}$

$>$ assessing people with eating and drinking difficulties

$>$ dietary advice and modification

$>$ clinically-assisted nutrition and hydration

$>$ the law

$>$ ethical decision making

$>$ guiding practice.

\section{Assessing people with eating and drinking difficulties}

Multiple factors determine our ability to eat and drink successfully, including the pre-oral and oral preparatory phases, the act of swallowing, airway protection, any underlying disease, and the caring environment. These key determinants highlight the complexity and vulnerability of oral nutrition and hydration; impairment in any one of these areas will affect the ability of a person to eat and drink (Fig 1)

To gain a complete understanding of an eating and drinking problem, clinicians will need to assess four main areas of clinical practice:

> the pre-oral phase of eating and drinking, intra-oral preparation of food and fluid, and swallowing

> laryngeal closure, cough and pulmonary function

$>$ the underlying medical, neurological, surgical and psychiatric conditions

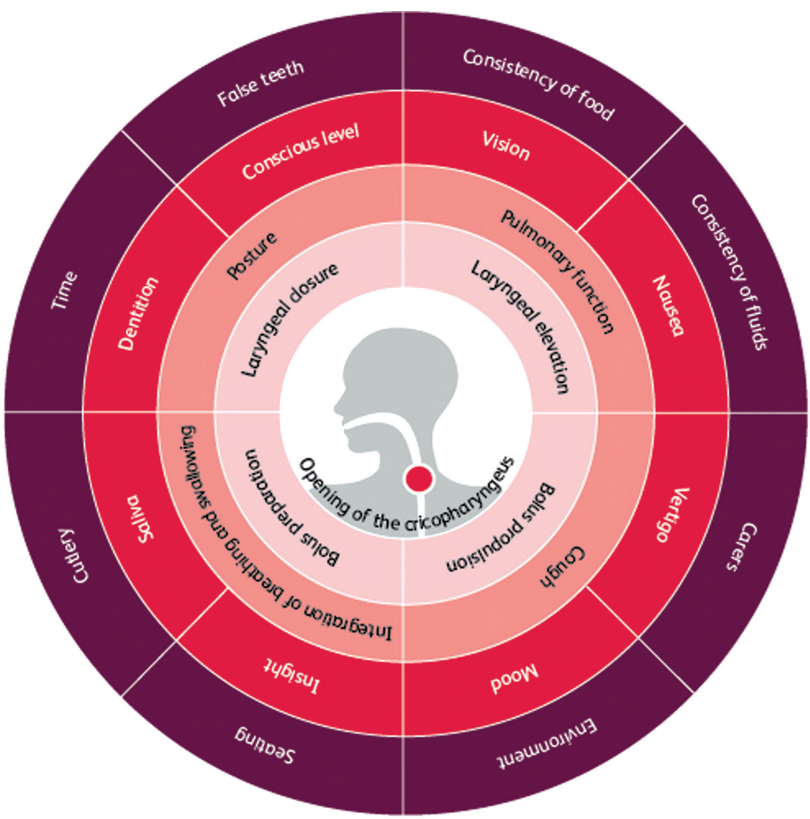

Fig 1. Some of the key determinants of successful eating and drinking.
$>$ the environment, including the availability of carers and the consistency of the available food and fluids.

The consultation between a health professional and a patient with eating and drinking difficulties (real or perceived) usually relates to three key questions.

$>$ What is the underlying diagnosis?

$>$ What is the mechanism of the eating and drinking problem?

$>$ Can the person eat and drink and, if so, at what risk?

The diagnosis of the underlying disease is made in the usual way, based on the history, examination and investigations. The mechanism of the problem with eating and drinking can usually be inferred from the diagnosis and is informed by the findings of relevant investigations such as videofluoroscopy and fibreoptic endoscopic evaluation (FEES).

Assessing the ability to eat and drink (and judging the associated risks) is informed by a knowledge of the underlying disease and an understanding of the exact mechanism of the problem, but may also require repeated observation using appropriate quantities and consistencies of food and drink over a representative time period.

\section{Dietary advice and modification}

Eating and drinking are not simply essential for maintaining nutrition and hydration. They are important for pleasure and social interactions; food, drinks and mealtimes are a way in which we connect with others and can often be an expression of our cultural identity.

It is therefore important to make every effort to maintain and support these activities. The provision of oral nutritional support (which can include food fortification, use of nourishing drinks and oral nutritional supplements) should be the preferred method of choice for any patient with inadequate food and fluid intake to meet requirements, unless they cannot swallow safely, have inadequate gastrointestinal function or if no benefit is anticipated, for example in the last days of end-of-life care. ${ }^{4,5}$

During the last year of life, screening for malnutrition using a validated screening tool such as the Malnutrition Universal Screening Tool (MUST) or the Patients Association Nutrition Checklist can be helpful in identifying nutritional problems at the earliest opportunity, so that action can be taken to reverse or slow down nutritional deterioration. ${ }^{6,7}$

Effective strategies to support eating and drinking to optimise nutritional intake involve multidisciplinary team working together with good communication with patients, family members and carers. The aims of oral nutritional support should take into account the stage of disease and prognosis. Dietary advice should be tailored to ensure that it remains a benefit, not a burden. Patients and their carers should be offered advice on how to manage specific difficulties with eating and drinking, perhaps due to side effects or symptoms associated with a disease or its treatment. It is important to consider the patient's social, physical, psychological, clinical and cultural needs, as well as their religious beliefs (Fig 2).

National evidence-based pathways (such as www. malnutritionpathway.co.uk) can improve the use of food-based oral nutritional strategies and oral nutritional supplements. Local resources will also guide management of malnutrition in acute trusts and in primary care. General guidance should support, 


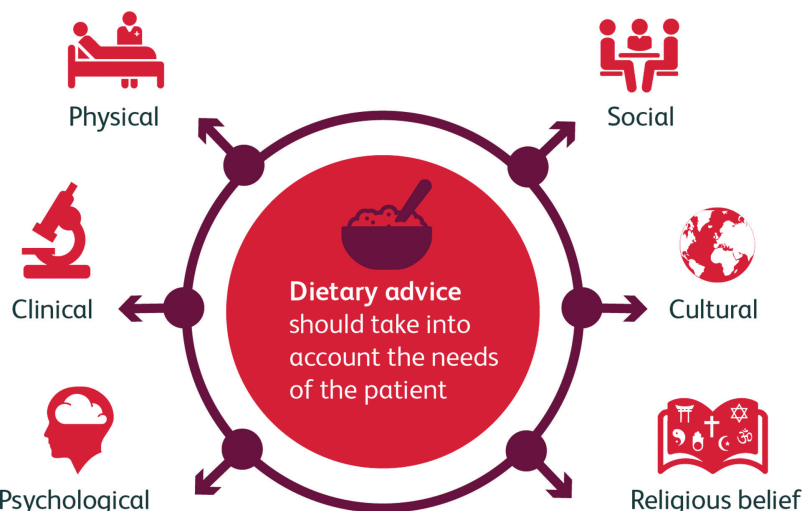

Fig 2. The factors impacting on dietary advice.

rather than replace, clinical judgement and individual assessment undertaken by skilled practitioners.

Sensory techniques, positioning and postural strategies, texture modifications and adaptations of food and fluids, and behavioural and cognitive techniques are all tools to support people to continue to eat and drink orally. Dietitians and speech and language therapists (SLTs) can provide advice on texture-modified diets, and educate and train others in care settings to provide safe, patientcentred nutritional care.

The International Dysphagia Diet Standardisation Initiative (Fig 3) was developed to provide a framework, common terminology and descriptions for the consistency of foods and fluids on a worldwide basis. ${ }^{8}$ Changes in both portion and bolus size are important. Medication may also have to be prescribed in alternative forms, requiring liaison between doctors, pharmacists, SLTs and dietitians. ${ }^{4}$

The term 'risk feeding' has been used to refer to individuals who continue to eat and drink orally despite a perceived risk of choking

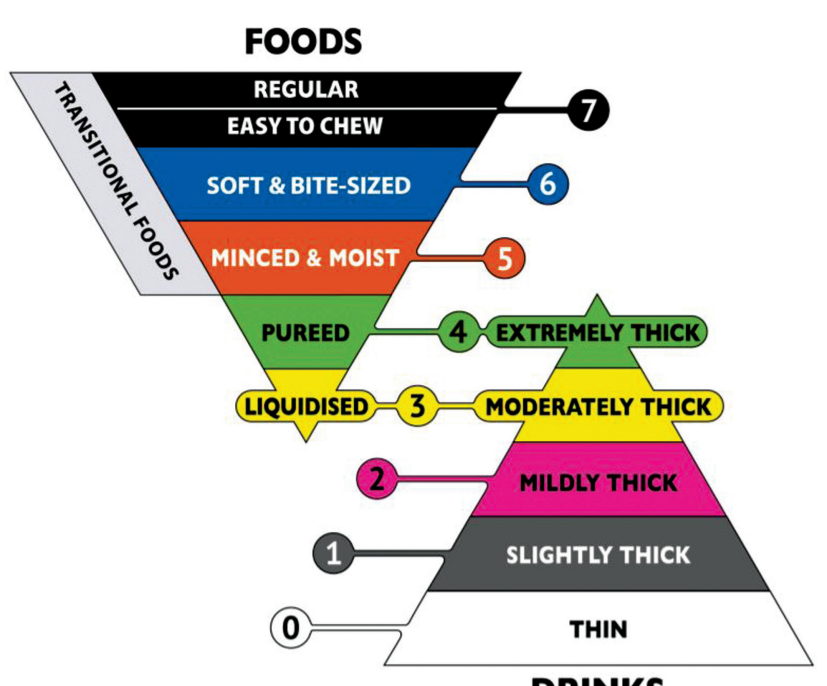

DRINKS

Fig 3. The International Dysphagia Diet Standardisation Initiative. Reproduced with permission from The International Dysphagia Diet Standardisation Initiative 2019 (https://iddsi.org/framework). or aspiration. 'Risk feeding' may occur in a range of situations, such as:

> when someone with capacity makes a decision to eat and drink despite the risk

> where $\mathrm{CANH}$ is not appropriate or declined

$>$ where the benefits of eating and drinking orally (such as enjoyment and enhanced quality of life) are deemed to outweigh the risks (such as chest infections or choking).

'Risk feeding' decisions should be made carefully, balancing the risks and safety with a person's quality of life. The decision process must comply with relevant law and professional guidance. Policies should seek to mitigate unnecessary delays in providing food, fluid and medications and undesirable restrictions of oral intake.

\section{Clinically-assisted nutrition and hydration}

Clinically-assisted nutrition and hydration (CANH) refers to all forms of artificial nutrition support, including tube feeding and parenteral nutrition. Guidance produced in 2018 by the British Medical Association (BMA) and RCP has created a framework for clinicians and healthcare workers to help guide decision making. ${ }^{9}$ CANH should be considered for people who are malnourished or at risk of malnutrition and have inadequate or unsafe oral intake.

Decisions to use CANH can be complex and should carefully consider individual circumstances; patients and their families should be supported by a multidisciplinary team. Whether to start, continue or withdraw nutrition and hydration towards the end of life remains a difficult and contentious issue.

Enteral tube feeding should be considered in patients with inadequate or unsafe oral intake with a functional and accessible gastrointestinal tract. Tube feeding should be stopped when the patient is re-established on adequate oral intake. It is vital that all healthcare organisations have policies and training in place to support the safe practice for enteral tube feeding, given that misplaced tubes and other tube-related complications can lead to serious harm or death. Parenteral nutrition should only be considered in those with a non-functional, inaccessible, short, obstructed or perforated gastrointestinal tract. A multidisciplinary nutrition support team should assess all patients considered for parenteral nutrition to ensure that this modality of feeding is appropriate and safe. ${ }^{4}$

A nutrition support team should assess all patients referred for gastrostomy placement, and consideration of the risks versus benefits is key. Gastrostomy placement is invasive with significant morbidity and mortality, particularly in older people and those with debilitating disease. Patients nearing the end of life may prefer to take in food and fluid orally and enjoy the taste, even though intake may be suboptimal. The patient should always be at the centre of clinical decision making and the focus should be on reaching the best decision for that person.

There is no good evidence to support gastrostomy feeding in people with advanced dementia, and studies suggest that it should only occur in exceptional circumstances. ${ }^{10}$ Following gastrostomy insertion, patients with advanced dementia have significantly higher mortality than those without dementia. ${ }^{11}$ In general, careful support with oral nutrition and hydration is preferred.

As people approach the end of life (and ideally before starting $\mathrm{CANH}$ ), the appropriateness of continuing enteral or parenteral nutrition support and intravenous fluids should be considered. 
Clear reasons should be identified and recorded for withdrawal of nutrition and hydration.

At this stage, the provision of compassionate care is paramount. Frequent attendance of healthcare staff at the bedside is important to support the patient and the family and ensure that they do not feel abandoned, especially if nutrition and hydration have been withdrawn. ${ }^{12}$

\section{The law}

For adults, the two key concepts in decisions around medical treatment are capacity and best interests. This also applies to $\mathrm{CANH}$.

When patients have capacity, the guiding principle should be that of autonomy, which involves the right of the patient to refuse treatment. An adult patient with capacity has the right to decline even life-preserving treatment. They do not, however, have the right to demand any treatment which the healthcare team does not consider is clinically appropriate.

The Mental Capacity Act (MCA) 2005 (applicable in England and Wales) is the legal framework for determining mental capacity (Box 1) and making decisions on behalf of people who lack the capacity to decide for themselves.

Where someone has been assessed as lacking capacity to make a specific decision regarding their care and treatment, section 5 of the MCA provides legal protection for a clinician to carry out an act in their best interests. This includes both the giving and the withholding of treatment. ${ }^{14}$

The Supreme Court has confirmed that it is lawful to give treatment only if it is in the patient's best interests. Accordingly, if the treatment is not in the patient's best interests, then it would be unlawful to give it, and therefore lawful to withhold or withdraw it. ${ }^{15}$

When a hospital accepts a patient, it has a duty to take reasonable steps to keep them alive; a duty which arises both in common law and in Article 2 of the European Convention on Human Rights. ${ }^{16}$ These reasonable steps include the provision of food and fluid with clinical assistance where required. Where a patient with capacity has expressed a wish to be kept alive, deliberately withholding this treatment would be unlawful.

There may be circumstances in which clinicians consider that the provision of food and fluid is not clinically appropriate because the patient is dying and the priority is to allow them to die with dignity and free from pain. In the event that discussions with the patient or (where the patient lacks capacity) those interested in their welfare do not produce agreement as to the way forward, then the clinicians should seek legal advice as to how to proceed.

In determining best interests, healthcare professionals must look at a patient's welfare in the widest sense; not just medical, but social and psychological. They must consider the nature of the medical treatment, what it involves and its prospects of success. They must ask what the patient's attitude to the treatment is or

\section{Box 1. Determining capacity ${ }^{13}$}

The Mental Capacity Act says that a person has capacity if they are able to:

$>$ understand the information relevant to the decision

$>$ retain that information

$>$ use or weigh that information as part of the process of making the decision

$>$ communicate their decision.
Box 2. Determining best interests

To determine a patient's best interests, the Mental Capacity Act says that healthcare professionals should:

$>$ not discriminate or make assumptions on the basis of the person's age, appearance, condition or behaviour

> consider whether the person will at some time regain capacity and, if this is likely, whether the decision could be postponed

$>$ encourage participation by doing whatever is possible to permit or encourage the person to take part

> not be motivated by a desire to bring about the person's death where the decision relates to life-sustaining treatment

$>$ consider all the relevant circumstances by trying to identify the things the person lacking capacity would take into account if they were making the decision themselves

$>$ find out the person's views, including their past and present wishes and feelings, and any beliefs or values that might influence their decision if they had capacity; this should include consulting family, carers and anyone granted a lasting power of attorney.

would be likely to be; and they must consult others for their view of what his or her attitude would be. ${ }^{17}$

Section 4 of the MCA sets out a series of steps that must be taken to determine whether a decision or action is in a person's best interests. These are described in Box 2.

Advance decisions (also known as advance directives or living wills) to refuse particular medical treatments are recognised in common law and are legally binding. Where an advance decision applies to the treatment in question, a healthcare professional would be civilly and criminally liable if they deliberately carried out treatment in the face of it. Adequate steps should always be taken to investigate precisely the terms of an advance decision. ${ }^{18}$

Almost all dilemmas in the law of consent are resolved by healthcare professionals in consultation with the patient and their family. Healthcare professionals must ensure that they follow the provisions of the MCA as well as the relevant guidance in the code of practice. However, if at the conclusion of a medical decisionmaking process in relation to life-sustaining treatment, there remain concerns about the way forward, a difference of opinion or a lack of agreement, then an application to the Court of Protection must be made. This is an inalienable right of the individual, guaranteed by the European Convention on Human Rights.

The nature of decision making in relation to children and young people under 18 years will depend upon whether they are aged $16 / 17$ years or aged 15 years or below. Gillick competence states that children under 16 years should be regarded as able to make the decision if they are believed to have enough understanding and intelligence to appreciate what is involved. Consent to treatment can be obtained from a Gillick competent child aged under 16 years, from a person with parental responsibility in the case of a child under 16 years lacking Gillick competence, or from the court. If they are aged $16 / 17$ years then the same presumption of capacity applies as for an adult, but if they lack capacity, then in most cases either section 5 of the MCA can be relied upon or consent from a person with parental responsibility.

\section{Ethical decision making}

If the patient does not have capacity and cannot make their own decisions, then their voice will have to be relayed by others. The 
decision-making process should seek to come to the decision that is as far as possible aligned to the decision that person would make if they had capacity to make it. Where no advance decision applies, best interests decision making should be constructed around the known wishes and feelings of the person lacking capacity.

This collection of information and opinions should be respectful, unhurried and comprehensive, as well as carefully documented. Important principles to guide these decisions and discussions are the sanctity of life and the preservation of dignity. These principles apply whether the patient has capacity or not.

The principle of the sanctity of life does not mean that life should be preserved at all costs. For instance, it need not oppose withdrawal or withholding of treatment in particular cases. Neither does the principle of the sanctity of life mean that a patient's autonomous wish to refuse treatment may be overridden. This ethical view is consistent with English law, which recognises the sanctity of life but does not treat it as an absolute principle, as stated in the Bland case. ${ }^{19}$

This must be considered against the background of rightful patient expectations that they receive good clinical care, that they are included in decisions about their care and that they are treated with dignity. Healthcare professionals should be aware that 'good' for a patient may be a complex notion that cannot be reduced to a single ethical dimension and may not coincide with their own point of view.

The patient, or their representatives, should understand that $\mathrm{CANH}$ is a burdensome treatment with risks. A consensus of ethical opinion and legal precedent is clear that CANH constitutes a medical treatment, rather than basic care. As such, ethically and legally, it can be withheld or withdrawn if it is thought not to be in the patient's best interests.

Transparency, honesty and respect should guide discussions around CANH. Every case is different and there is not one correct answer which fits all situations. It may be helpful to seek additional professional opinions (eg from other experienced healthcare professionals, a local ethics forum or legal representatives).

In ethical terms, there is a distinction between killing and letting die. Medicine, law and everyday morality distinguish clearly between a strong universal prohibition on killing and a more equivocal attitude to letting die. Withholding CANH may permit the patient's underlying condition to progress to the end of life. This is not the same as killing.

\section{Guiding practice}

Decisions to start, continue or withdraw nutrition and hydration are challenging and can be a major source of anxiety for clinicians and patients. The final chapter in the guidance is intended as a tool to inform and guide such decisions. Fig 4 summarises the key factors that impact on those decisions. In most cases there are no easy answers, but rather approaches to follow to reach the best decision for each individual patient.

The guidance includes a number of illustrative examples of patients to help guide practice. ${ }^{2}$ These cover a broad range of conditions and circumstances to explore specific difficulties and dilemmas. Each one is followed by key questions to consider relating to supporting people with eating and drinking and best interests, to give practical guidance on what to consider and the appropriate steps to follow. The examples cover the risks versus benefits of a gastrostomy, whether to carry out 'ad hoc' wishes

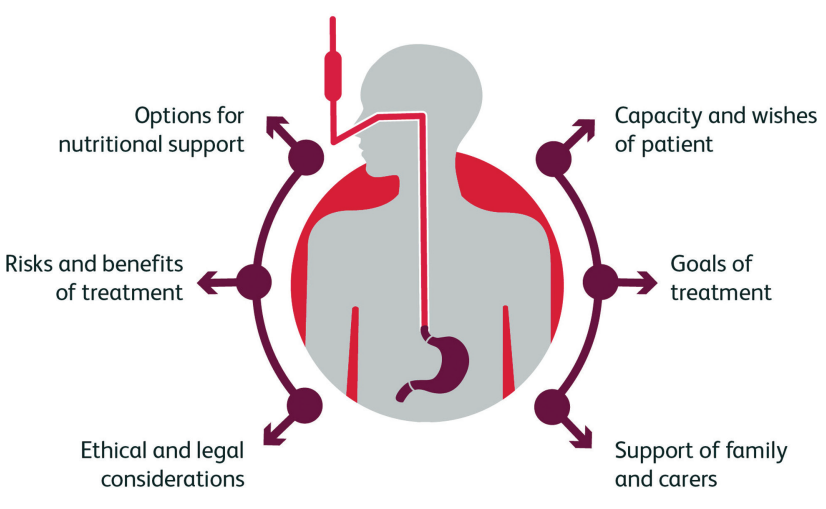

Fig 4. Key factors impacting on nutrition support decisions.

expressed by a patient, assessing capacity, determining best interests, assessing quality of life and when parenteral nutrition should be offered. Box 3 provides a truncated illustrative example.

Box 4 offers a number of important questions to ask in relation to all patients with eating and drinking difficulties regarding the benefits of treatment. These are explored more fully in the guidance. $^{2}$

\section{Conclusion}

In summary, all patients requiring nutrition and hydration support should receive coordinated care from a multidisciplinary team.

People should be supported to continue to eat and drink via oral means unless they cannot swallow safely, have inadequate gastrointestinal function or if no benefit is anticipated. CANH should be considered for people who are at risk of malnutrition and have inadequate or unsafe oral intake. However, gastrostomy placement is invasive with significant morbidity and mortality, particularly in older people and those with debilitating disease. It is not recommended in people with advanced dementia.

The benefits of any intervention should outweigh the risks. The risks versus benefits should be clearly documented and treatment goals articulated.

An adult patient with capacity has the right to decline even lifepreserving treatment. The MCA sets out the legal framework for determining mental capacity and making decisions on behalf of people who lack the capacity to decide for themselves.

Whether to start, continue or withdraw nutrition and hydration towards the end of life remains a difficult and contentious issue. The patient should always be at the centre of decision making and the course of action or inaction should be the result of careful, measured consideration and what is believed to be in the best interests of the individual patient.

\section{Conflicts of interest}

Nicola Burch is a member of the British Association for Parenteral and Enteral Nutrition (BAPEN) Medical Committee and RCP representative on the Royal College of Speech and Language Therapists 'Feeding at Risk' Advisory Group.

Chris Danbury is chair of the Legal and Ethical Policy Unit of the Faculty of Intensive Care Medicine and sits on the Royal College of Anaesthetists Ethics Committee. 
Box 3. Kwame, a stroke patient

Kwame, an 83-year-old man who lives alone, has had a sudden right hemiplegia and is aphasic. A formal speech and language therapy assessment has identified Kwame as having a high risk of aspiration with any oral intake. Nasogastric tube (NGT) feeding was tried. However, Kwame repeatedly pulled out the NGT, so the multidisciplinary team decided to insert a nasal loop which allowed it to stay in longer. At 2 weeks, Kwame was referred for a gastrostomy. His relatives report that when his sister had a stroke, he had said, 'You must shoot me if I am ever like that.' Currently, he lacks capacity to consent to any procedure. Should Kwame have a gastrostomy?

The natural history of eating and drinking problems after a stroke will inform this decision. Serial assessments and the elapsing of time following an acute neurological event allow the potential for recovery to be estimated. Usually a patient is fed by an NGT for 10-14 days as an interim measure before a gastrostomy is considered. Insertion of a gastrostomy tube after 14 days reduces mortality and improves nutritional outcomes at 6 weeks compared with continued nasogastric feeding. ${ }^{10}$ A proportion of patients will regain swallowing function within the first 2 weeks; at 4 weeks $20 \%$ of patients will no longer require tube feeding. ${ }^{20,21}$

Gastrostomy tube placement should not be an emergency procedure and should always be undertaken as a planned elective procedure, following clinical optimisation, in accordance with the patient's best interests, and with the agreement of the multiprofessional team. Pressure to place a gastrostomy early just to help facilitate faster discharge from hospital should be resisted.

Ad hoc or 'off the cuff' comments that do not specifically pertain to personal circumstances can result in an extremely emotive reaction from relatives. The facts surrounding such comments need to be explored and the clinical similarities or differences and/or origin of the concerns ascertained. While these views should be factored into best interests discussions and clinical assessments, they do not have any legal bearing on decision making if everyone is clear that they were off the cuff and not related to the person's own circumstances. Circumstances and opinions may also change and therefore too much weight should not be placed upon ad hoc comments made historically.

Patients may refuse treatment through an advance decision which is legally binding. An advance decision to refuse life-sustaining treatment has to be written, witnessed and clear that it applies even when life is at risk. Even if there is no advance decision to refuse treatment, if the patient's views can be ascertained with sufficient certainty, they should generally be followed or afforded great respect and will generally prevail over the very strong presumption in favour of preserving life. If a gastrostomy is not in the patient's best interests or goes against the patient's refusal (if they have capacity), inserting the gastrostomy tube could give rise to a charge of assault.

Simon Gabe is a member of NHS England clinical cell related to parenteral nutrition group, BAPEN and British Society of Gastroenterology.

Anne Holdoway has received honoraria for speaking engagements from Nutricia Ltd, Danone, Abbott Nutrition, and Fresenius-Kabi, and an unrestricted educational grant from Nutricia to support the work of the 'Managing disease-related adult malnutrition in the community' national expert panel and fund a website for a number of commercial organisations. All work is related to nutrition, diet and nutrition support in chronic disease.

\section{Box 4. Key questions regarding the benefits of} treatment

Is clinically-assisted nutrition and hydration (CANH) necessary for adequate nutrition and hydration or can sufficient intake to cover basic needs be taken orally, albeit with difficulty?

Does the patient have capacity to decide upon the method of receiving nutrition and hydration? If not, what decision is in their best interests and in accordance with their wishes (if known)?

If CANH is necessary, what is the best method/route for giving it?

Do the benefits of treatment outweigh the burdens/risks of treatment?

What are the goals of the nutrition and hydration support, the expected duration, and the criteria for stopping?

With whom should discussions about the future management be held?

What are the key issues to discuss with the patient, relatives, carers, healthcare workers and advocates?
Jeremy Nightingale is chair of the British Intestinal Failure Alliance (BIFA) and co-chair of the Nightingale Trust for Nutritional Support. He is chair of the adjudication committee for a phase III efficacy and safety trial of apraglutide (VectivBio AG).

Andrew Rochford is regional clinical director for NHS England and Improvement (Emergency Care Improvement Support Team); and member of the Executive Board and Council of BAPEN.

Trevor Smith is president and chair of the Board of Trustees of BAPEN.

Aminda De Silva is a member of the British Society of Gastroenterologists and a member of BAPEN. He has accepted honoraria from several different pharmaceutical companies to allow him to attend medical conferences as well as for speaking/ chairing symposia engagements.

\section{References}

1 Care Policy and Evaluation Centre at the London School of Economics and Political Science. Projections of older people with dementia and costs of dementia care in the United Kingdom 2019-2040. CPEC working paper 5. London: CPEC, 2019.

2 Royal College of Physicians. Supporting people who have eating and drinking difficulties. A guide to practical care and clinical assistance, particularly towards the end of life. Report of a working party. London: RCP, 2021. www.rcplondon.ac.uk/projects/outputs/ supporting-people-who-have-eating-and-drinking-difficulties [Accessed 11 May2021].

3 Royal College of Physicians. Oral feeding difficulties and dilemmas: A guide to practical care, particularly towards the end of life. Report of a working party. London: RCP, 2010.

4 National Institute for Health and Care Excellence. Nutrition support for adults: oral nutrition support, enteral tube feeding and 
parenteral nutrition: Clinical guideline [CG32]. NICE, 2017. www.nice.org.uk/guidance/cg32 [Accessed 11 May 2021].

5 Baldwin C, Weekes CE. Dietary counselling with or without oral nutritional supplements in the management of malnourished patients: a systematic review and meta-analysis of randomised controlled trials. J Hum Nutr Diet 2012;25:411-26.

6 MUST calculator. www.bapen.org.uk/screening-and-must/mustcalculator [Accessed 11 May 2021].

7 Patients Association Nutrition Checklist. www.patients-association. org.uk/patients-association-nutrition-checklist-toolkit [Accessed 11 May 2021].

8 International Dysphagia Diet Standardisation Initiative. IDDSI, 2019. www.iddsi.org [Accessed 11 May 2021].

9 British Medical Association and Royal College of Physicians. Clinically-assisted nutrition and hydration (CANH) and adults who lack the capacity to consent. Guidance for decision-making in England and Wales. BMA, 2018. www.bma.org.uk/media/1161/ bma-clinically-assisted-nutrition-hydration-canh-full-guidance.pdf [Accessed 11 May 2021].

10 Volkert D, Chourdakis M, Faxen-Irving G et al. ESPEN guidelines on nutrition in dementia. Clin Nutr 2015;34;1052-73.

11 Sanders DS, Carter MJ, D'Silva J et al. Survival analysis in percutaneous endoscopic gastrostomy feeding: a worse outcome in patients with dementia. Am J Gastroenterol 2000;95:1472-5.

12 Brody H, Campbell ML, Faber-Langendden K, Ogle KS. Withdrawing intensive life sustaining treatment - recommendations for compassionate clinical management. New Engl J Med 1997;336:652-57.
13 Mental Capacity Act 2005, section 3(1). www.legislation.gov.uk/ ukpga/2005/9/contents [Accessed 11 May 2021].

14 Aintree University Hospitals NHS Foundation Trust v James [2013] UKSC 67 at paragraphs 20 and 22.

15 NHS Trust $v$ Y [2018] UKSC 67 at paragraph 92.

16 R (Burke) v General Medical Council \& Ors [2006] QB 273.

17 Aintree University Hospitals NHS Foundation Trust v James [2013] UKSC 67 at paragraph 39

18 See by analogy, NHS Cumbria CCG v Rushton [2018] EWCOP 41.

19 Airedale NHS Trust $v$ Bland [1993] AC 789 House of Lords.

20 Westaby D, Young A, O'Toole P, Smith G, Sanders DS. Guidelines. The provision of a percutaneously placed enteral tube feeding service. Gut 2010;59:1592-1605.

21 James A, Kapur K, Hawthorne AB. Long-term outcome of percutaneous endoscopic gastrostomy feeding in patients with dysphagic stroke. Age Ageing 1998;27:671-6.

Address for correspondence: Karen Porter, Royal College of Physicians, 11 St Andrews Place, Regent's Park, London NW1 4LE, UK.

Email: karen.porter@rcp.ac.uk 